\title{
Novos desafios da educação a distância: programação e uso de Chatbots
}

\author{
New challenges in distance learning: programming and use of Chatbots
}

\author{
Daniela Melaré Vieira Barros* \\ Aníbal Martins Guerreiro
}

\section{Resumo}

A evolução tecnológica, particularmente a Inteligência Artificial, tem contribuído significativamente para mudanças relevantes em quase todas as dimensões da nossa sociedade, nomeadamente na educação e, consequentemente, no ensino a distância. Tais mudanças exigem transformações significativas, especialmente no processo de ensino-aprendizagem, de forma a minimizar a incapacidade de os Tutores Virtuais responderem na totalidade às solicitações de suporte por parte dos alunos e, por isso, a necessidade enorme de potenciar as tecnologias existentes, com o objetivo de minorar essa dificuldade. Este artigo tem como objetivo compreender os novos desafios da educação a distância $(\mathrm{EaD})$ no ensino superior on-line, nomeadamente a programação e o uso de Chatbots, bem como as razões e causas para sua operacionalidade. A metodologia utilizada foi baseada no método dedutivo, resultante do processo de pesquisa bibliográfica e documental, em consonância com a investigação de doutoramento em curso sobre a temática dos tutores virtuais. Como resultado, a literatura especializada tem destacado a enorme vantagem do uso de Chatbots (assistentes virtuais) na EaD no ensino superior on-line, devido aos enormes benefícios para os intervenientes diretos do processo de ensino-aprendizagem, ou seja: a instituição, os tutores e os alunos.

Palavras-chave: Chatbot. EaD. Inteligência-Artificial. Novos desafios. Programação. Tutores virtuais.

\section{Abstract}

The technological evolution has contributed significantly to relevant changes in almost all dimensions of our society, namely in education and, consequently, Distance Learning (EaD). Such changes require significant transformations, particularly in the teaching-learning process, and in order to respond to the inability of Virtual Tutors to respond fully to all requests for support from the students and therefore, there is an enormous need to strengthen technologies in order to minimize this problem. This article aims to understand the new challenges of Distance Education (EaD) in online higher education, namely the programming and use of Chatbots, as well as the reasons and causes for its operation. The methodology used was based on the deductive method, resulting from the bibliographic and documentary research process. As a result, the specialized literature has highlighted the enormous advantage of the use of Chatbots (virtual assistants) in Higher Education in online education, due to the enormous benefits to the direct participants of the teaching-apprentice process, that is: The institution, the students.

Keywords: Chatbot. EaD. Artificial Intelligence. New challenges. Programming. Virtual Tutors.

Recebido em 13/10/2018 - Aprovado em 12/12/2018

http://dx.doi.org/10.5335/rep.v26i2.8743

Professora Auxiliar, Universidade Aberta, Portugal. E-mail: daniela.barros@uab.pt

Doutorando na Universidade Aberta, Portugal. E-mail: anibalmguerreiro@gmail.com 


\section{Introdução}

A educação a distância (EaD) é uma das modalidades de ensino que mais tem crescido no panorama nacional e internacional, nomeadamente nas economias emergentes, como a Índia e a China. Utilizando como suporte a internet e as tecnologias associadas, como a Inteligência Artificial (IA) e a Computação Cognitiva (CG), entre outras, disponibiliza um conjunto de recursos potencialmente mais eficazes para o sucesso do processo de ensino-aprendizagem. Neste contexto, a Instituição de Ensino necessita fazer os ajustamentos adequados às competências pedagógicas, tecnológicas e outras, ou até mesmo adaptar as novas realidades ao modelo pedagógico da instituição, de forma a potenciar ao máximo todos os recursos disponíveis para o sucesso dos alunos, mas nem sempre isso acontece.

É objetivo deste artigo a apresentação de uma nova abordagem para aumentar a rapidez e o suporte aos alunos e potenciar o uso das tecnologias no processo de ensino-aprendizagem: a programação e o uso de um Chatbot (Assistente Virtual) na EaD. Neste artigo, será abordada a problemática e os conceitos relacionados com os Tutores Virtuais, a Inteligência Artificial e a Realidade Virtual. Além disso, será também discutida a inserção dos Chatbots na educação.

A programação dos Chatbots nos processos educativos é extremamente importante porque resolve uma série de problemas com que as instituições de ensino superior a distância se deparam, entre eles: a personalização do atendimento, o ajuste dos conteúdos a cada processo de aprendizagem como uma ajuda constante e rápida e, sobretudo, o acompanhamento do desempenho de cada estudante. Os Chatbots, além de permitirem essa personalização, a redução de tempo e custos, podem funcionar também em vários tipos de plataformas, facilitando, assim, as aplicações técnicas.

Esta tecnologia (que não é nova) tem sido aplicada com sucesso em muitos setores da nossa sociedade, nomeadamente, em áreas como o Marketing Digital, Indústria Financeira, Recursos Humanos, Comércio Eletrónico, Saúde e Turismo. Dadas as suas características, começou também a ser usada com êxito no ensino presencial e a distância, sendo os casos mais conhecidos, respetivamente, o projeto Jill Watson, na Georgia Tech College of Computing - USA e, ultimamente, o caso da The Genie of Deakin University - Australia, que serão detalhados mais à frente neste artigo.

$\mathrm{O}$ aumento exponencial dos cursos superiores de EaD, e dos cursos MOOCs, (Massive Open Online Courses) é uma realidade em muitos países. De acordo com Goel e Polepeddi (2016), mais de cinquenta e oito milhões de estudantes em todo o mundo acederam a estes cursos. Estes cursos necessitavam de tutores para trabalhar o feedback e as relações de aprendizagem, porém o número de tutores não au- 
mentou na mesma proporção, o que fez com que o apoio aos alunos sofresse alguma falta de eficácia e demorasse mais tempo a ser dado. Ainda de acordo com os mesmos autores, a eficácia da aprendizagem em muitos MOOCs pode ser questionável, uma vez que a permanência dos alunos nestes cursos é normalmente inferior a $50 \%$.

Embora haja várias razões para a baixa permanência de estudantes nos cursos, a principal razão é a falta de interatividade ou a necessidade de maior feedback e interação nos contextos da educação on-line e a distância (GOEL; POLEPEDDI, 2016; BIDAISEE, 2017; WALLACE; ICE; GIBSON, 2011; KHALIL; EBNER, 2014). Assim, uma das principais recomendações para melhorar a eficácia da aprendizagem nos MOOCs e, portanto, também na melhoria da retenção de estudantes, é melhorar a interação entre o professor e os alunos (HOLLANDS; TIRTHALI, 2014). Neste contexto, este artigo traz a discussão e a análise sobre a seguinte pergunta: Para além dos motivos clássicos de dificuldades de apoio aos tutores, como tempo, condições técnicas e dificuldades de comunicação e habilidades de empatia pedagógica, de que forma é que um Chatbot (Tutor Virtual) poderá facilitar e contribuir por um apoio rápido e eficiente aos estudantes?

\section{Orientações metodológicas do estudo}

A metodologia utilizada neste artigo foi baseada no método dedutivo, resultante do processo de pesquisa bibliográfica e documental sobre os temas aqui tratados. $\mathrm{O}$ estudo apresentado é parte do projeto de doutoramento em desenvolvimento e tem como base a pesquisa exploratória e o desenvolvimento do estado da arte sobre o tema da tutoria virtual.

As opções metodológicas deste estudo basearam-se numa significativa diversidade de estratégias de recolha de informação, privilegiando o recurso a metodologias qualitativas, tendo em conta o objeto do estudo. Devido à dimensão do trabalho, que limita a introdução de elementos de informação complementares, que poderiam conduzir a processos de triangulação de dados, assim como uma maior contextualização dos estudos, optou-se por designá-lo apenas por estudo.

A realização do estudo aqui descrito teve como sustentação teórica as referências do modelo pedagógico da Universidade Aberta (PEREIRA et al., 2007) e as fundamentações que sustentam os aspetos pedagógicos da educação a distância, como Garrison (1989), Aretio, Corbella e Figaredo (2007), Anderson (2008), Garrison e Anderson (2003) e Downes (2007). Com base nas referências da tutoria virtual, foi possível melhor compreender esta importante área da $\mathrm{EaD}$, através dos autores Alves, Cabral e Costa (2003), Amarilla (2011), Sangrà et al. (2011), Belloni (2008) e 
Barros e Reis (2009). Em relação aos Chatbots, as evidências apontadas pelos autores Muldowney (2017), Gomes (2017), Zumstein e Hundertmark (2017), Srdanovic (2017) e Schappo (2017), permitiram abordar diferentes aspetos da implementação e da arquitetura de um Chatbot e compreender o potencial do seu uso na EaD.

\section{EaD: a função tutorial}

A EaD é mais do que um conjunto de alunos e tutores comunicando-se entre si através das tecnologias, é um conjunto de elementos (Instituição, Qualidade, Avaliação, Modelo Pedagógico, Infraestruturas, Curso/Currículo, Professor, Tutor, Tecnologias e o Aluno), todos interligados e trabalhando em conjunto para que 0 sucesso coletivo se verifique. Neste contexto, poderemos considerar o diagrama a seguir como uma visão geral da $\mathrm{EaD}$, em que os diversos componentes se apresentam como camadas, que devem ser lidas do interior para o exterior. $\mathrm{O}$ centro de todo o processo é o aluno, tudo é centrado nele. $\mathrm{O}$ aluno está diretamente ligado ao seu Curso, que é baseado num currículo, que é orientado pelo tutor/professor, cuja base está no uso das tecnologias. O processo de ensino-aprendizagem é ministrado nas Infraestruturas da Instituição e baseado no Modelo Pedagógico. A avaliação da qualidade da EaD estará sempre presente numa Instituição de Ensino.

Figura 1 - Diagrama representando os principais componentes da EaD

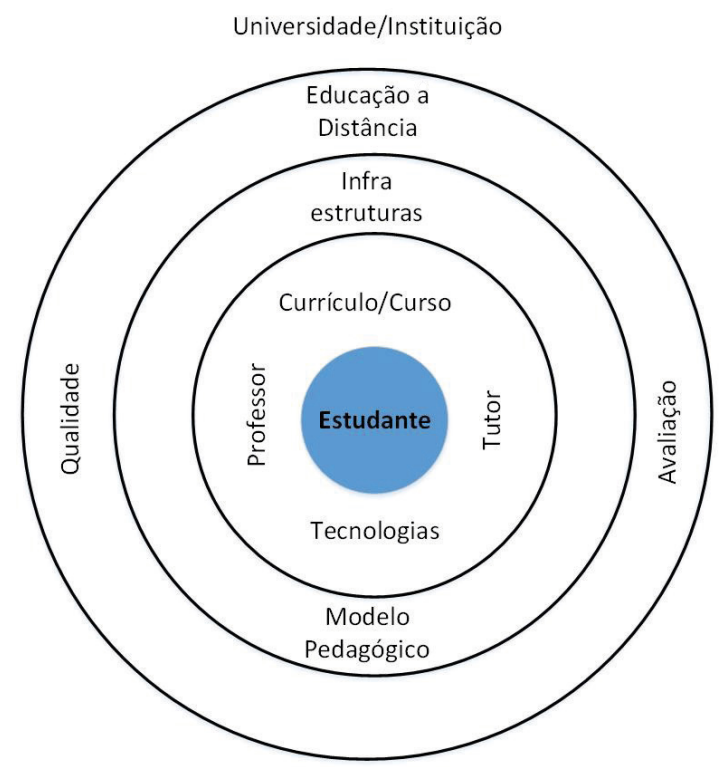

Fonte: elaborado pelo autor (BELLONI, 2008; MORGADO, 2003; MOORE; ANDERSON, 2003; ALVES; CABRAL; COSTA, 2003). 
Alves, Cabral e Costa (2003) sumarizam as características do EaD da seguinte forma: separação professor-aluno: o docente não está presente em termos físicos, mas transmite os seus conhecimentos, faz as suas planificações e organiza o que 0 aluno vai aprender; utilização de meios tecnológicos: a utilização de recursos tecnológicos de comunicação e interação, tais como: videoconferência, áudios, vídeos, computadores ligados em rede, e-mail, entre outros, estão na base tecnológica do $\mathrm{EaD}$; organização de apoio (tutoria): a atuação do tutor (orientador da aprendizagem do aluno) é extremamente importante na $\mathrm{EaD}$, sendo que este pode trabalhar a distância, individualmente ou em pequenos grupos, mas orientando sempre os alunos na direção dos seus estudos; aprendizagem independente e flexível: através do EaD procura-se, além de transmitir conhecimentos, tornar o aluno capaz de "Aprender a Aprender" e "Aprender a fazer", de forma flexível, respeitando a sua autonomia em relação ao tempo, estilo, ritmo e método de aprendizagem; comunicação multidirecional: no EaD, o aluno não funciona apenas como um recetor de informação, pode também ter uma participação ativa, quer respondendo às perguntas que lhe foram feitas, quer participando ativamente no fórum de discussão; educação massiva: as novas tecnologias aumentam a possibilidade de trocas de materiais educativos, eliminando fronteiras espaço temporais e propiciando o seu aproveitamento por um grande número de pessoas.

O ensino a distância, usando como suporte a internet e todas as tecnologias associadas, oferece novas formas de aprender e de ensinar, que romperam com os paradigmas do ensino tradicional, alterando e inovando os modelos pedagógicos, democratizando o ensino e flexibilizando o acesso a pessoas que, por razões várias, estavam excluídas do ensino presencial.

As instituições educativas não substituíram as instituições de ensino presencial, contudo, devido às vantagens que oferecem e à oferta que apresentam, a procura pelos seus cursos é enorme. Nunes $(2009$, p. 2-8) refere que o acesso às Universidades Abertas, por todo o mundo, está a aumentar de uma forma significativa e "o crescimento vertiginoso da demanda por matrículas é o calcanhar-de-Aquiles do ensino presencial". Costa (2016), no seu artigo "Tendências contemporâneas em educação superior a distância no mundo e no Brasil", refere que o EaD é a modalidade de ensino que tem apresentado maior crescimento no cenário da educação superior internacional, salientando que, no Brasil, as matrículas entre 2003 e 2013 nos cursos superiores aumentaram cerca $2.300 \%$.

Leal e Gouveia (2015, p. 197-206) referem que a grande procura de cursos a distância está "causando alguma agitação em várias instituições escolares, sobre- 
tudo universitárias, devido sobretudo à quantidade de participantes que nos cursos mais populares atingem dezenas de milhares de participantes".

Amarilla (2011) defende que a educação a distância implica uma dicotomia entre as tarefas dos processos de ensinar (estrutura organizacional, planeamento, conceção metodológica, produção de materiais) e dos processos de aprender (características e necessidades dos estudantes, modos e condições de estudos, níveis de motivação, etc.).

Um grupo de investigadores da Universidade Aberta da Catalunha construiu uma definição moderna e inclusiva de e-learning, que é aceite pela maioria da comunidade científica e como referência para estudiosos e profissionais da área. A definição a que chegaram foi que o ensino a distância é:

[...] una modalidad de enseñanza y aprendizaje, que puede representar todo o una parte del modelo educativo en el que se aplica, que explota los medios y dispositivos electrónicos para facilitar el acceso, la evolución y la mejora de la calidad de la educación y la formación (SANGRÀ et al., 2011, p. 5).

O sucesso da EaD está relacionado com a relação biunívoca entre os tutores e os alunos, onde os recursos pedagogicamente consistentes e disponíveis atempadamente podem fazer a diferença entre sucesso e insucesso. A EaD é um espaço educativo importante, cujo desenvolvimento é essencialmente apoiado pelas tecnologias e pelo tutor virtual. De acordo com Belloni (2008, p. 15), “[...] esse profissional desempenha diversas atividades docentes e de mediador, que podem passar pela elaboração de materiais didáticos e de suporte à aprendizagem, esclarecimento de dúvidas e correção de trabalhos".

Etimologicamente, tutor vem do latim tutore, protetor, no entanto, a palavra tutor tem tido vários significados ao longo da história e, muitas vezes, dependo das circunstâncias em que a palavra é usada, o seu significado muda. No contexto educacional, um tutor está presente em universidades ou colégios e consiste numa pessoa envolvida na gestão da informação e outras funções. Estas incumbências são também chamadas: "tutoria", "tutorial" ou "tutorial": nela, o tutor observa os problemas dos estudantes e ajuda, prestando assistência de forma mais célere, eficaz e imediata (FERREIRA, 1986).

A evolução tecnológica permitiu, também, ir além dos ambientes virtuais de aprendizagem, onde estes funcionam como suporte e gestão de conteúdos, e através dos tutores, interpretes dos objetivos do curso, é possível utilizar outras formas de fazer chegar o conhecimento aos alunos. É reconhecido pela literatura da área que os tutores, com o decorrer dos tempos, têm vindo a ganhar importância na EaD, assumindo tarefas essenciais no processo de ensino-aprendizagem. 
Segundo Mauri Collins e Zane Berge (1996 apud MACHADO; MACHADO, 2004), as tarefas dos tutores classificam-se em quatro áreas: pedagógica, gerencial, técnica e social. De acordo com Arguis (2002 apud NOFFS; TORRES, 2014), apresenta de uma forma mais resumida as três características que um tutor deve possuir: qualidades humanas (a empatia, a sociabilidade, a responsabilidade e a capacidade de aceitação); qualidades científicas (conhecimento da maneira de ser do aluno, dos elementos pedagógicos para conhecer e ajudar o aluno) e qualidades técnicas (trabalhar com eficácia e em equipe e participando em projetos).

As análises de Barros e Reis (2009) apresentam as funções, habilidades e perfil do tutor da EaD, de uma forma simples e abrangente, na Tabela 1.

Tabela 1 - Funções, competências, habilidades e perfil do tutor da EaD

\begin{tabular}{|c|c|c|c|}
\hline Função & Competência & Habilidade & Perfil \\
\hline $\begin{array}{l}\text { Ter uma cultura tecnológica para } \\
\text { facilitar a sua comunicação e a in- } \\
\text { terface com os alunos. }\end{array}$ & $\begin{array}{l}\text { Em cultura } \\
\text { tecnológica. }\end{array}$ & $\begin{array}{l}\text { Uso de aparelhos digitais, comu- } \\
\text { nicar-se pelas tecnologias digi- } \\
\text { tais com os alunos, domínio de } \\
\text { uso dos aparelhos da tecnologia } \\
\text { em geral, compreensão da lógica } \\
\text { de uso dos aparelhos digitais. }\end{array}$ & $\begin{array}{l}\text { Domínio da infor- } \\
\text { mática. }\end{array}$ \\
\hline $\begin{array}{l}\text { Ter o domínio do computador e } \\
\text { compreensão geral do seu funcio- } \\
\text { namento. }\end{array}$ & $\begin{array}{l}\text { No uso dos apli- } \\
\text { cativos básicos } \\
\text { do computador. }\end{array}$ & $\begin{array}{l}\text { Uso avançado dos aplicativos do } \\
\text { word, excel e power point, capa- } \\
\text { cidade de conectar os periféricos } \\
\text { do computador e resolver peque- } \\
\text { nos problemas técnicos. }\end{array}$ & $\begin{array}{l}\text { Domínio da infor- } \\
\text { mática. }\end{array}$ \\
\hline $\begin{array}{l}\text { Estabelecer um espaço com o do- } \\
\text { cente responsável pela disciplina } \\
\text { para a troca de informações peda- } \\
\text { gógicas e as dificuldades que pos- } \\
\text { sivelmente poderão ser sanadas no } \\
\text { processo de ensino-aprendizagem. }\end{array}$ & $\begin{array}{l}\text { Na área } \\
\text { pedagógica. }\end{array}$ & $\begin{array}{l}\text { Troca de informações e procura } \\
\text { de informações necessárias so- } \\
\text { bre os temas. }\end{array}$ & $\begin{array}{l}\text { Disponibilidade } \\
\text { Responsabilidade. }\end{array}$ \\
\hline $\begin{array}{l}\text { Ter consciência dos aspetos éticos } \\
\text { que envolvem a sua função em re- } \\
\text { lação aos alunos e ao docente do } \\
\text { curso. }\end{array}$ & Ética e moral. & $\begin{array}{l}\text { Análise e julgamento baseados } \\
\text { em princípios morais, éticos que } \\
\text { norteiam toda e qualquer prática } \\
\text { em sua vida e não somente a } \\
\text { profissional. }\end{array}$ & Responsabilidade. \\
\hline $\begin{array}{l}\text { Atualizar-se constantemente na área } \\
\text { à qual pertence. }\end{array}$ & $\begin{array}{l}\text { Em iniciativa na } \\
\text { busca da forma- } \\
\text { ção continuada. }\end{array}$ & $\begin{array}{l}\text { Organização da vida pessoal, } \\
\text { profissional e académica, de ma- } \\
\text { neira que a formação continuada } \\
\text { seja constante e natural. }\end{array}$ & $\begin{array}{l}\text { Independência } \\
\text { Disponibilidade } \\
\text { Iniciativa } \\
\text { Organização } \\
\text { Ser estudioso. }\end{array}$ \\
\hline $\begin{array}{l}\text { Exercer o papel de motivador do } \\
\text { aluno, que por problemas diversos } \\
\text { e pessoais pode apresentar dificul- } \\
\text { dades no cumprimento das ativida- } \\
\text { des propostas. }\end{array}$ & $\begin{array}{l}\text { Em motivar } \\
\text { fazendo uso } \\
\text { das palavras. }\end{array}$ & $\begin{array}{l}\text { Escrever de forma empática e } \\
\text { motivadora. }\end{array}$ & $\begin{array}{l}\text { Capacidade de } \\
\text { inferência } \\
\text { Criatividade } \\
\text { Empatia. }\end{array}$ \\
\hline
\end{tabular}




\begin{tabular}{|c|c|c|c|}
\hline $\begin{array}{l}\text { Acompanhar os alunos, auxiliando } \\
\text { nas dúvidas académicas, burocráti- } \\
\text { cas e gerais do curso ou disciplina } \\
\text { ao qual está vinculado. }\end{array}$ & $\begin{array}{l}\text { Em conhecimen- } \\
\text { to dos processos } \\
\text { de gestão do } \\
\text { curso em todos } \\
\text { os aspetos. } \\
\end{array}$ & $\begin{array}{l}\text { Conhecimento de toda a estrutu- } \\
\text { ra administrativa e legislativa do } \\
\text { curso. }\end{array}$ & \begin{tabular}{|l|} 
Disponibilidade \\
Organização \\
Ser estudioso \\
Curioso e argu- \\
mentativo. \\
\end{tabular} \\
\hline $\begin{array}{l}\text { Esclarecer dúvidas quanto ao } \\
\text { conteúdo da disciplina ou curso, } \\
\text { enviando, se necessário, material } \\
\text { complementar. }\end{array}$ & $\begin{array}{l}\text { Na área ou con- } \\
\text { teúdo do curso. }\end{array}$ & $\begin{array}{l}\text { Organização didática do conteú- } \\
\text { do, reelaborando o material com } \\
\text { qualidade e focando, em espe- } \\
\text { cial, no aluno que apresenta di- } \\
\text { ficuldades. }\end{array}$ & $\begin{array}{l}\text { Responsabilidade } \\
\text { Empatia } \\
\text { Criatividade } \\
\text { Disponibilidade } \\
\text { Ser tolerante e } \\
\text { ao mesmo tempo } \\
\text { exigente. }\end{array}$ \\
\hline $\begin{array}{l}\text { Argumentar, aos questionamentos, } \\
\text { dúvidas e inferências, com respon- } \\
\text { sabilidade, dentro dos prazos pro- } \\
\text { postos. }\end{array}$ & $\begin{array}{l}\text { Em capacidade } \\
\text { argumentativa. }\end{array}$ & $\begin{array}{l}\text { Responder e estabelecer um } \\
\text { processo de diálogo com os } \\
\text { alunos, cumprindo com os com- } \\
\text { promissos assumidos de forma } \\
\text { responsável. }\end{array}$ & $\begin{array}{l}\text { Responsabilidade } \\
\text { Organização } \\
\text { Independência } \\
\text { Disponibilidade } \\
\text { Iniciativa. }\end{array}$ \\
\hline $\begin{array}{l}\text { Procurar antecipar-se às necessi- } \\
\text { dades dos alunos, a partir das ca- } \\
\text { racterísticas pessoais de cada um. }\end{array}$ & $\begin{array}{l}\text { Em relaciona- } \\
\text { mento interpes- } \\
\text { soal. }\end{array}$ & $\begin{array}{l}\text { Perceber as necessidades do } \\
\text { outro de maneira empática, faci- } \\
\text { litando a resolução do problema } \\
\text { de forma criativa. }\end{array}$ & $\begin{array}{l}\text { Empatia } \\
\text { Criatividade. }\end{array}$ \\
\hline $\begin{array}{l}\text { Encaminhar dúvidas ou questões e } \\
\text { procurar ajuda nos momentos ne- } \\
\text { cessários, compartilhando informa- } \\
\text { ção e conhecimento. }\end{array}$ & $\begin{array}{l}\text { Em trabalho } \\
\text { multi e } \\
\text { masinterdisci- } \\
\text { plinar }\end{array}$ & $\begin{array}{l}\text { Trabalhar em equipe de maneira } \\
\text { colaborativa. }\end{array}$ & $\begin{array}{l}\text { Responsabilidade } \\
\text { Disponibilidade } \\
\text { Iniciativa } \\
\text { Flexibilidade } \\
\text { Ser Curioso e } \\
\text { Argumentativo. }\end{array}$ \\
\hline $\begin{array}{l}\text { Saber organizar o tempo de acesso } \\
\text { ao ambiente virtual de ensino, de } \\
\text { maneira a desenvolver as ativida- } \\
\text { des com qualidade. }\end{array}$ & $\begin{array}{l}\text { Em organização } \\
\text { temporal. }\end{array}$ & $\begin{array}{l}\text { Priorizar tarefas importantes e } \\
\text { urgentes com eficácia e asserti- } \\
\text { vidade. }\end{array}$ & $\begin{array}{l}\text { Organização } \\
\text { Criatividade } \\
\text { Independência } \\
\text { Disponibilidade } \\
\text { Iniciativa. }\end{array}$ \\
\hline
\end{tabular}

Fonte: Barros e Reis (2009).

A Tabela 1 sumariza, de uma forma clara, as funções, as habilidades e o perfil do tutor da EaD, fazendo a respetiva associação e mostrando a relação existente entre elas em cada linha da tabela.

Autores como Koehler e Mishra (2009) defendem uma estrutura conceptual denominada Technology, Pedagogy, and Content Knowledge (TPACK), pois referem que este modelo é útil para os tutores quando começam a usar ferramentas e estratégias digitais, para apoiar o ensino e a aprendizagem. Este modelo é projetado em torno da ideia de que o conteúdo (o que você ensina) e a pedagogia (como você ensina) devem ser a base para qualquer tecnologia que se planeia usar na sala de aula para melhorar a aprendizagem. O framework fornece um mapa que permite mostrar como se pode integrar a tecnologia na sala de aula, de forma eficaz. Este modelo indica os níveis de conteúdo, pedagogia e potencial tecnológico que cada 
professor demonstra na sua prática letiva, com base no uso das tecnologias. De acordo com os autores, os professores/tutores precisam ser competentes nos três domínios.

Ainda nesta linha de pensamento, em relação aos tutores, é necessário considerar que "[...] não basta ele possuir o domínio de sua área de conhecimento e dos recursos tecnológicos, é preciso também procurar desenvolver habilidades e estratégias pedagógicas para atender a um público diverso" (BELLONI, 2008, p. 50).

Tendo em vista os factos mencionados, somos levados a acreditar que os docentes desempenham múltiplas funções, tornando-os imprescindíveis no processo de ensino-aprendizagem. Considerando o aumento exponencial da procura dos cursos em educação a distância, coloca uma enorme pressão de mudança constante nas instituições de ensino superior. As mudanças que se impõem terão que ser forçosamente ao nível de todos os intervenientes no processo de ensino-aprendizagem, nomeadamente ao nível das funções dos tutores e dos modelos pedagógicos.

Neste cenário, existe a necessidade de as instituições disporem de tutores multifacetados de forma contínua, interativa, personalizada e à conveniência dos alunos. Para além disso, com acesso de forma regular aos conteúdos, ao feedback e ao apoio nas suas áreas de estudo, juntando, ainda, o que podemos pontuar como vantagens de um assistente virtual ou Chatbot.

Deixamos claro neste trabalho que os Chatbot são um apoio complementar ao trabalho da tutoria, serão potencializadores do processo, ampliando as capacidades de rapidez e atendimento mais detalhado e personalizado dos estudantes, de acordo com objetivos e competências a serem desenvolvidas.

Uma das limitações do uso de um Chatbot é o facto de ele poder não fazer atividades para as quais foi "programado". A operacionalização das suas tarefas depende de um conjunto de regras, inseridas dentro de um algoritmo, que são seguidas de uma forma rigorosa e, por isso, quando surge alguma situação imprevista, ele pode ficar "perdido" e confuso e, por conseguinte, dar informações fora do contexto da situação, ou seja, falta-lhe capacidade de improvisação e de compreensão. Eles não são humanos, por isso, ainda não é possível os Chatbots capturarem, na totalidade, as variantes de uma conversa humana.

Apesar dos Chatbots poderem usar a inteligência artificial e, consequentemente, poderem "aprender" a ter comportamentos similares aos humanos, não é fácil incorporar sentimentos como, por exemplo, empatia, ironia, sarcasmo, alegria, tristeza ou mesmo humor. Este obstáculo faz com que não exista imprevisibilidade e que se distancie da humanização pretendida. Outra limitação é a dificuldade 
de encontrar especialistas na área, pois, apesar da facilidade na sua criação e desenvolvimento, certos projetos podem exigir um elevado grau de conhecimento em várias áreas e tornar-se extremamente complicado o seu desenvolvimento, ter custos muito altos e ser muito demorado o seu avanço.

\section{Programação e os Chatbots: conceito e características}

O que é um Chatbot? Chatterbot (ou Chatbot) é um programa de computador que tenta simular um ser humano na conversação com as pessoas. O objetivo é responder às perguntas de tal forma que as pessoas tenham a impressão de estar conversando com outra pessoa e não com um programa de computador. Após o envio de perguntas em linguagem natural, o programa consulta uma base de conhecimento e, em seguida, fornece uma resposta que tenta imitar o comportamento humano (GOMES, 2017).

Os Chatbots são utilizados em várias áreas de negócio (como o Siri, que é um Chatbot exclusivo da Apple que usa o processamento de linguagem natural para responder a perguntas, fazer recomendações e executar ações, ou o Alexa, que é um serviço de voz da Amazon que permite criar uma forma mais intuitiva de interagir com a tecnologia que os utilizadores usam diariamente.) e funcionam como um novo canal de informação, comunicação e transação de informação que permite que as empresas cheguem ao seu público-alvo através de software como Facebook, WhatsApp ou WeChat (ZUMSTEIN; HUNDERTMARK, 2017).

Grande parte dos Chatbots têm como base a Inteligência Artificial (IA), pois a IA possibilita que eles aprendam através de um padrão nos dados, e isto está a torná-los mais reais que nunca, aumentando a sua capacidade com os humanos de uma forma mais natural, eficaz e inteligente.

A inteligência artificial abrange um grande leque de áreas de atuação, entre elas: o conhecimento; o raciocínio; a resolução de problemas; a perceção; a aprendizagem, o planeamento e a capacidade de manipular e mover objetos.

A inteligência artificial engloba várias áreas de ação, entre elas a Realidade Virtual, definida como uma forma avançada de interface, onde o utilizador pode ter a sensação de estar dentro de um ambiente tridimensional. "Ela é capaz de oferecer uma sensação real de viver em um ambiente criado pelo computador, sentir e tocar objetos que não existem" (LEITE; BRAGLIA; PEREIRA, 2011, p. 4). Quando o utilizador entra neste mundo tridimensional, encontra uma cópia da realidade e 
pode interagir totalmente com este ambiente, utilizando equipamentos especiais, tais como capacete, luvas, controle, entre outros (RUSSELL; NORVIG, 1995).

As características dos Chatbots variam de acordo com o tipo e, consequentemente, com a área de atuação. Em relação ao tipo, os Chatbots podem classificar-se em: baseados em regras e baseados em IA. No que concerne aos Chatbots baseados em regras, a sua ação é muito limitada e não têm capacidade de aprendizagem, pois não podem atuar fora das regras previamente definidas. Os Chatbots baseados em IA, por outro lado, aprendem com a sua atuação, pois podem responder a perguntas bastantes complexas.

De uma forma geral, os Chatbots, de acordo com Schappo (2017), devem estar disponíveis $24 \mathrm{~h}$ por dia, 7 dias por semana; deve existir uma automatização dos processos; devem existir múltiplos canais de atendimento e a possibilidade de entrar em contacto com seu Lead (utilizador com quem comunica) de uma forma automatizada. De acordo com Morgan (2017), a sua principal responsabilidade é simplificar as interações entre serviços e pessoas. Srdanovic (2017) argumenta que os Chatbots na EaD devem poder automatizar as classificações, permitir uma aprendizagem intervalada e integrar a funcionalidade de avaliação dos cursos por parte dos alunos. A capacidade de resposta às solicitações dos alunos de uma forma rápida e a capacidade de individualização do ensino são características muito importantes num Chatbot.

A Realidade Virtual, base referencial dos Chatbots, engloba várias áreas de atuação. No que respeita à educação, podemos considerar os Sistemas Tutores Inteligentes (STIs), que são uma evolução dos primeiros sistemas de aprendizagem nesta área, denominados sistemas CAI (Computer Aided Instruction), que, contrariamente às primeiras versões, já utilizam técnicas de Inteligência Artificial que procuram proporcionar uma experiência customizada de aprendizagem para o estudante, simulando as interações professor-aluno (LUSTOSA; ALVARENGA, 2004).

As análises de Martins e Guimarães (2012) referem que um ambiente virtual deve agregar as seguintes características: Sintético: significa que o ambiente é gerado em tempo-real por um sistema computacional; tridimensional: significa que o ambiente que cerca o utilizador é representado em três dimensões (3D); Multissensorial: significa que mais do que uma modalidade sensorial é usada para representar o ambiente, com o sentido visual, sonoro, espacial (de profundidade), de reação do utilizador com o ambiente; Imersivo: entende-se, aqui, mais do que olhar e ouvir um display vindo de um monitor, o "display" necessita de criar a impressão de que se está dentro do ambiente produzido computacionalmente (nor- 
malmente, um sistema imersivo é obtido com o uso de capacetes de visualização, mas outros Sentidos, como o som e controles reativos, são também importantes); Interativo: refere-se à capacidade do computador detetar as entradas do utilizador e modificar instantaneamente o mundo virtual e as ações realizadas sobre ele; Realístico: envolve a precisão com que o ambiente virtual reproduz os objetos reais, as interações com os utilizador e o próprio modelo do responsável por dar ao utilizador a impressão de que ele está fisicamente dentro do ambiente virtual.

Os Ambientes Virtuais de Aprendizagem (AVA) desenvolvidos para a EaD permitem a colaboração e a interação em tempo real entre professores e alunos de uma forma remota de caráter multidimensional, associando simultaneamente diversas tecnologias, como: Internet, webmail, fórum, portefólio, biblioteca, diários, editores colaborativos e chats (LEITE; BRAGLIA; PEREIRA, 2011). Kirner e Siscoutto (2007) acrescentaram ainda que, além da Realidade Virtual permitir um ambiente Colaborativo para a Educação, é ainda um ambiente multiutilizador, baseado na teoria pedagógica do construcionismo, funcionando na Internet para suporte a diferentes aplicações educacionais.

Neste contexto, verifica-se uma excelente simbiose entre os Chatbots e os ambientes virtuais de aprendizagem (AVA) propriamente ditos, na medida em que é possível associar a capacidade das simulações, do realismo e de todas as outras características dos AVA com o uso da inteligência artificial e a capacidade de aprendizagem (machine learning) dos Chatbots, apesar da sobreposição de algumas funcionalidades entre eles.

\section{Análises e reflexões sobre o estudo realizado}

Dadas as características da comunicação na $\mathrm{EaD}$, uma das formas que os Chatbots podem ser usados com sucesso é através de um sistema de perguntas e respostas, por meio do qual, um Chatterbot está pronto a comunicar com os estudantes e responder a todas as solicitações.

Um dos primeiros Chatbots a surgir foi o A.L.I.C.E (Artificial Linguistic Internet Computer Entity), um Chatterbot (ou Chatbot) criado na Lehigh University, por Richard S. Wallace, ativado em 1995, com capacidades de reconhecimento sonoro e interfaces gráficas que estimulam a comunicação entre a máquina e o homem (LEONHARDT; CASTRO; TAROUCO, 2003).

Outro exemplo de sucesso foi o Chatbot conhecido por "Jill Watson", criado pela "Georgia Tech - USA", que foi utilizado como tutor virtual durante um semes- 
tre no curso "Masters of Science in Computer Science", em que os alunos do curso não sabiam que "Jill” não era uma pessoa real. Segundo Lipko (2016), o professor Ashok Goel deu uma palestra no TEDx SanFrancisco sobre o uso de inteligência artificial e referiu que Jill Watson, a assistente de ensino da IA, foi baseado na plataforma Watson da IBM, que talvez seja mais conhecida como o computador que venceu dois campeões do "Jeopardy". "Jill" foi desenvolvido especificamente para lidar com o alto número de posts em fóruns de alunos matriculados no curso on-line.

Algumas universidades estão a adotar os Chatbots, que programam usando o sistema IBM Watson. Um desses exemplos é a Universidade Deakin, em Melbourne, Austrália, através de um dos seus projetos: "LIVE the Future" para aumentar a experiência estudantil. Este projeto de inovação com os Chatbots trouxe atenção internacional e aumentou as matrículas para 54.000 alunos em 2016 (LACITY et al., 2017).

O processo de construir a base de conhecimento do Chatterbot pode ser realizado/ programado pelo próprio aluno, pois existem ferramentas de autoria que facilitam a criação das definições a serem usadas no processamento da conversação, permitindo que o aluno tenha participação ativa no processo (LEONHARDT; CASTRO; TAROUCO, 2003). Dada a facilidade da programação dos Chatbots, conforme refere a literatura especializada, é aconselhável a sua implementação, em virtude do enorme retorno que esta tecnologia disponibiliza.

Uma das plataformas disponíveis, de uma forma gratuita e/ou paga, mais conhecidas para a criação/programação de Chatbots é a IBM Watson, cujo sistema se tornou famoso por ter vencido os campeões do programa televisivo americano Jeopardy (Quem quer ser milionário), em 2011. As áreas de atuação deste sistema são muito abrangentes, entre as quais se pode destacar: Advertising, Customer Engagement, Education, Financial Services, Health, IoT, Media, Talent e Work.

Um Chatbot pode ser criado com as funcionalidades descritas na Figura 2. 
Figura 2 - Funcionalidades para Chatbot

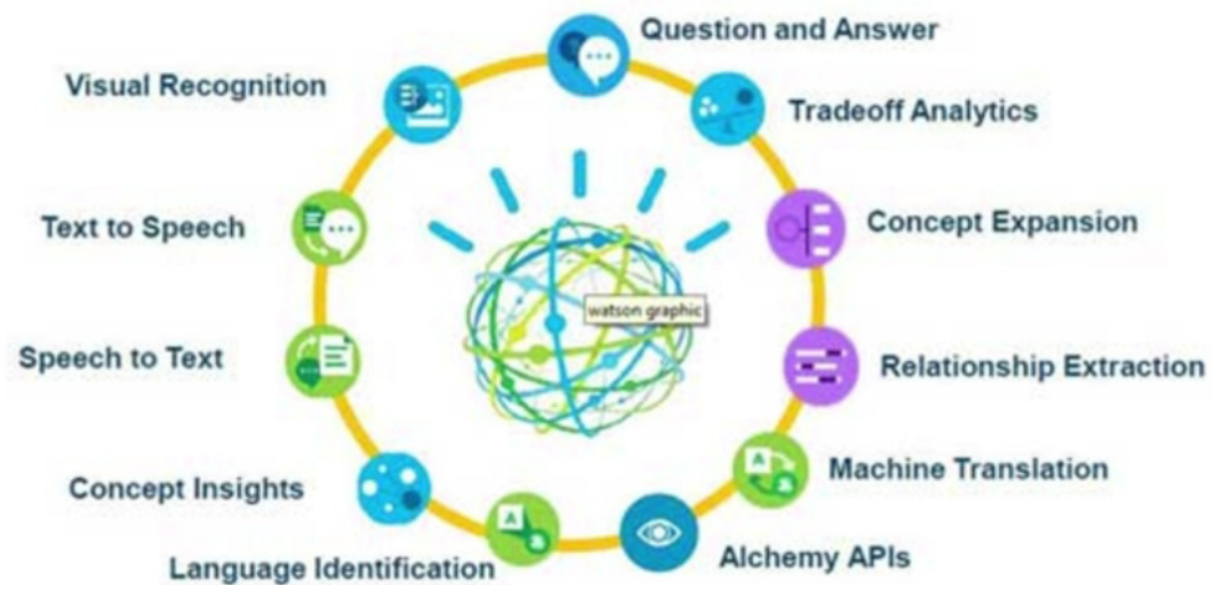

Fonte: disponível em: <https://www.ibm.com/watson/>. Acesso em: 22 set. 2018.

A Figura 3 representa a estrutura de um Chatbot em termos funcionais.

Figura 3 - Estrutura funcional de um Chatbot

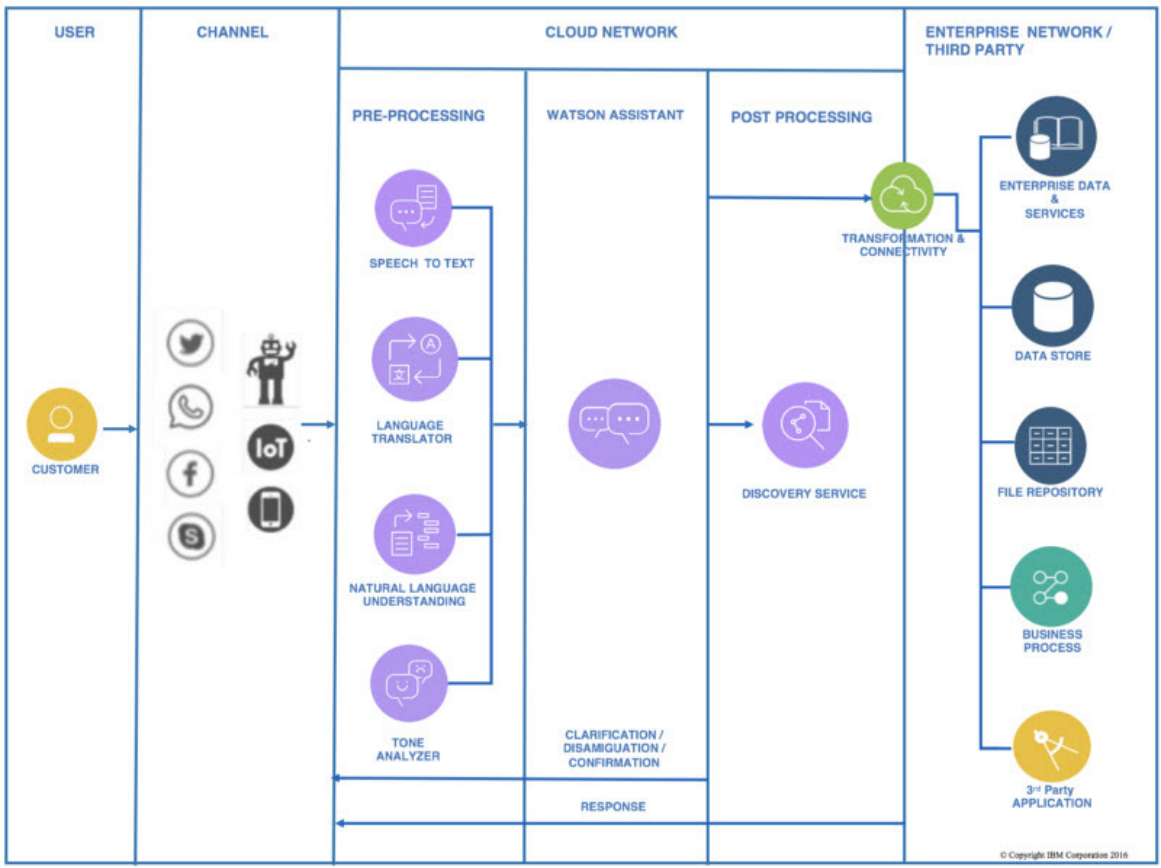

Fonte: disponível em: <https://www.ibm.com/watson/>. Acesso em: 22 set. 2018. 
Para usar o sistema IBM Watson (aplicação para criar Chatbots) são necessárias algumas etapas, nomeadamente o registo e a criação de uma conta, gratuita ou paga. Após concluída esta fase, para a criação de um Chatbot, é necessário escolher o tipo de aplicativo a criar e as suas funcionalidades (Figura 4). Para a criação de uma Assistente, as opções disponíveis na interface da aplicação são as representadas na Figura 4.

Figura 4 - Opções para o Assistente do Sistema IBM Watson

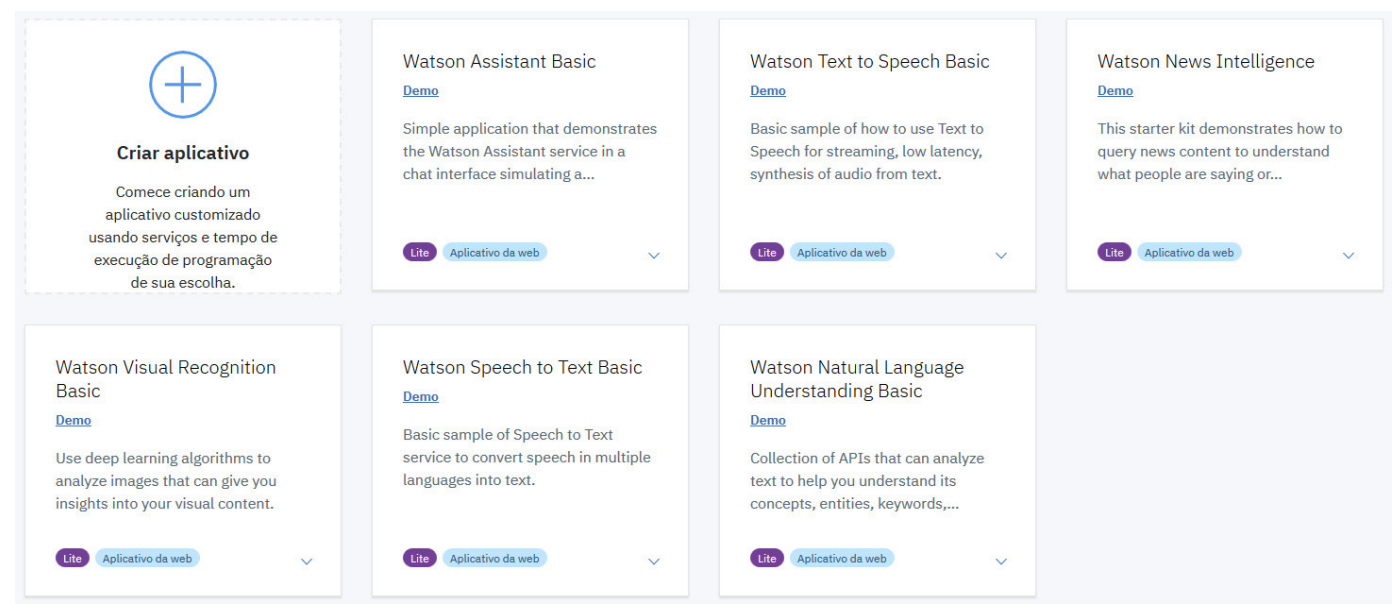

Fonte: disponível em: <https://www.ibm.com/watson/>. Acesso em: 22 set. 2018.

A Figura 5 representa o workingspace da aplicação IBM Watson Assistant, que permite a criação de uma forma mais ou menos rápida de Chatbots, os quais permitem realizar todas as operações necessárias para a construção de um aplicativo que permite a comunicação com humanos em linguagem natural, ou texto, simulando uma conversação entre pessoas. 
Figura 5 - Interface da aplicação para programar um Chatbot

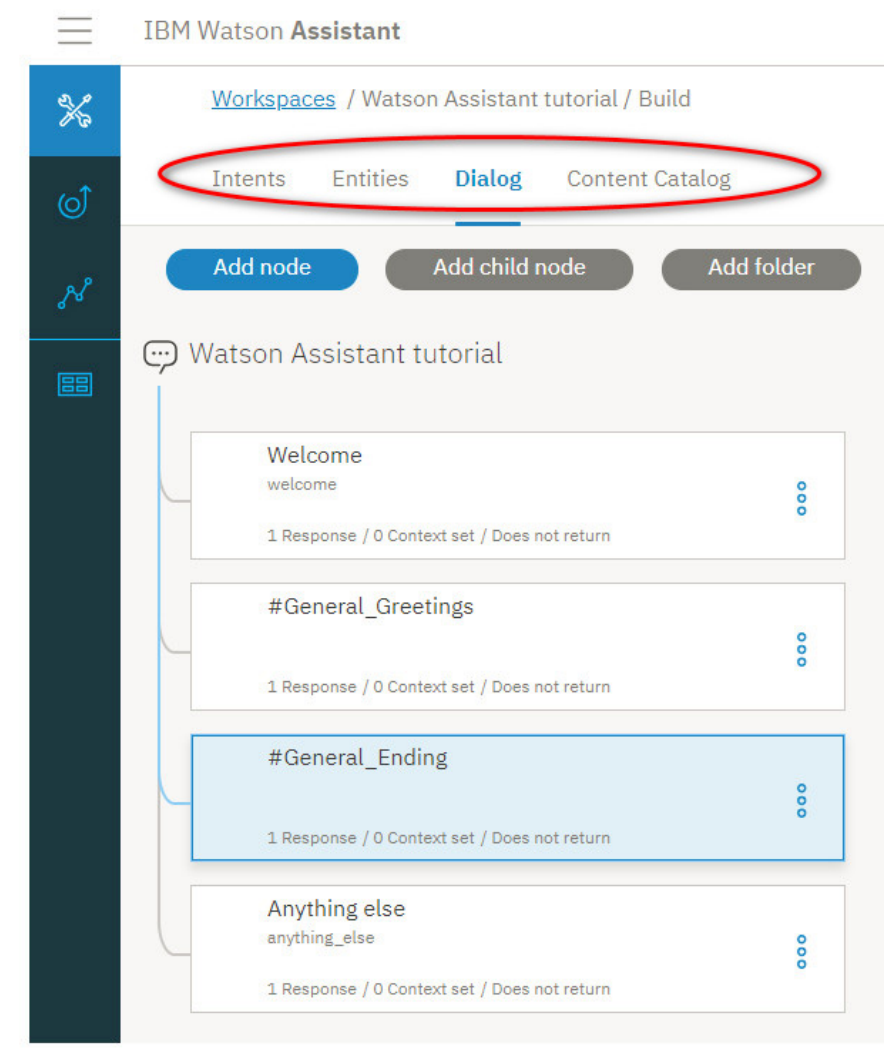

Fonte: disponível em: <https://www.ibm.com/watson/>. Acesso em: 24 set. 2018.

A criação de uma aplicação compreende as quatro componentes: Intents (Intenções), Entities (Entidades), Dialog (Diálogos) e Content Catalog (Catálogo de conteúdos).

As Intenções representam o objetivo do utilizador ou a razão da sua pergunta, por exemplo: Qual é a minha nota? É de referir que é aconselhável colocar no mínimo 7 Intenções, escritas de forma diferente, para que a "maquina" consiga entender o que o utilizador pretende. Por isso teria que ser usado pelo menos 7 sinónimos da pergunta: Qual é a minha nota? E poderiam ser: Qual é a minha classificação? Quantos pontos tenho no trabalho? etc.

As Entidades representam objetos que ajudam a clarificar as Intenções, que retratam os objetivos do utilizador. 
Os diálogos representam o "caminho" a percorrer pelo utilizador desde o início até à resposta final, verificando e procedendo de acordo com o reconhecimento (ou não) das Intenções/Entidades.

O catálogo de conteúdos fornece a forma mais fácil de adicionar intenções comuns à área de trabalho do Watson Assistant.

Neste exemplo, a seguinte figura, representa a interface do sistema IBM Watson, para a construção de Diálogos. Aqui estão apresentados os diálogos criados, relativamente à parte de Saudações, em que o sistema prevê um conjunto de sinónimos deste termo, para conseguir comunicar com os utilizadores. Se porventura, o utilizador introduzir um termo que não se coadune com os termos previstos nos referidos diálogos, o sistema, através da opção no diálogo: Anything else, "dirá" algo como: "Não compreendo o que quer dizer!".

Os Chatbots podem ser facilmente programados para realizar tarefas bastante complexas sem a necessidade de quem os programa ter conhecimento de linguagens de programação tradicionais. As suas potencialidades são reconhecidas por inúmeros estabelecimentos de ensino, sendo o Georgia Tech, nos EUA, o pioneiro no ensino presencial, com a famosa assistente Jill Watson, e a Universidade Deakin, Victoria, na Austrália, com o projeto Genie, ambos usando o sistema da IBM Watson.

Os Chatbots podem ser usados na EaD de várias formas, nomeadamente no fornecimento de informações sobre os cursos ou instituições de ensino, para aceitar inscrições dos alunos, podem ser utilizados no apoio na aprendizagem de conteúdos ou dar suporte/ajuda aos estudantes, funcionando como um verdadeiro assistente/ tutor.

Um dos projetos com mais reconhecimento a nível mundial foi a assistente Jill Watson, baseada no sistema da IBM Watson, criada em 2016, pelo professor Ashok Goel, do Georgia Tech Institute of Technology, que foi uma das 4 assistentes do curso de IA (3 humanos e a Jill Watson um Chatbot) com 3000 alunos, e que, durante um semestre, respondeu às perguntas colocadas pelos estudantes, sem que estes descobrissem que se tratava de uma "máquina" a responder às suas questões com um grau de certeza de 97\%. É de referir que a Jill Watson já está na $3^{\text {a }}$ versão e, num futuro próximo, estará disponível comercialmente para ser usada em instituições de ensino pelo mundo.

Outro exemplo do uso de Chatbots nas instituições de ensino é o Project Deakin Genie, assistente digital que interage com os estudantes no Campus da Universidade Deakin, que ganhou pelo segundo ano consecutivo o prémio: major Global Business Award. The Global Business Excellence Awards é um evento criado por 
uma organização Australiana independente que, através de um júri, atribui prêmios que visam ao reconhecimento, à competência e à excelência nas instituições publicas, privadas ou de caridade, em qualquer parte do mundo.

De acordo com Coyne (2017), Deakin Genie é uma plataforma de dispositivos móveis que integra Chatbots, inteligência artificial, reconhecimento de voz e um mecanismo de análise preditiva. Os alunos da Deakin University poderão, através de um/uma assistente virtual inteligente, aceder a tudo o que precisam no campus da Universidade. A plataforma de gestão de aprendizagem, a biblioteca digital, o sistema de suporte baseado no IBM Watson, entre muitos outros estão integrados na plataforma Genie.

Esta ferramenta destina-se a ajudar os alunos a acompanhar tudo o que está a acontecer, mantê-los motivados por meio de rastreadores de desempenho e ajudá-los a aproveitar ao máximo os materiais de aprendizagem e os serviços de suporte da Deakin.

A Figura 6 ilustra o diálogo entre o Chatbot e uma aluna em várias situações, sendo a primeira imagem o ecrã da primeira vez que a aluna entra no sistema, a segunda imagem a resposta pelo sistema à pergunta da aluna sobre as datas limites para entrega de trabalhos e a terceira imagem a organização de um trabalho de grupo.

Figura 6 - Interface do Ginie Deakin

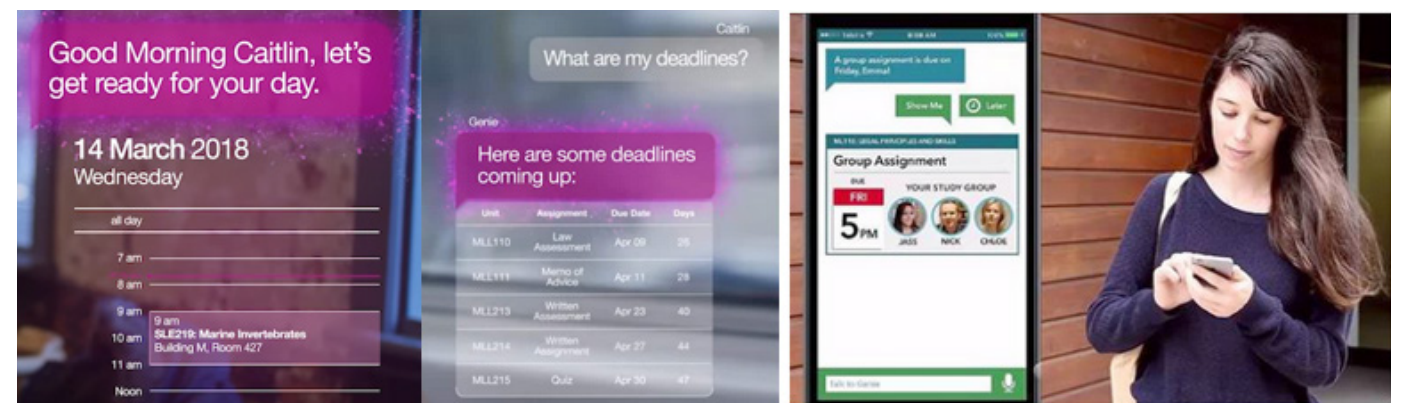

Fonte: disponível em: <https://www.itnews.com.au/news/meet-genie-deakin-unis-virtual-assistant-for-students-453230>. Acesso em: 24 set. 2018.

Dados os factos apresentados, é inegável a importância da utilização dos Chatbots na EaD, não só pela facilidade da sua criação, como também pelos benefícios que trazem à dinâmica entre tutores, alunos e às próprias instituições de ensino. As novas tecnologias emergentes e o uso da IA nos Chatbots têm con- 
tribuído para uma "humanização" e aprendizagem crescentes por parte destes aplicativos, permitindo uma comunicação quase "natural" entre o homem e a máquina. A proliferação dos Chatbots por todo o mundo é uma realidade, com uma sofisticação e eficiência cada vez maiores.

\section{Considerações finais}

A literatura especializada destacou a importância e os benefícios da programação de Chatbots na EaD. Dentro das evidências consideradas da pesquisa bibliográfica, destacaram-se alguns dos problemas com que as Instituições de Ensino Superior se deparam, nomeadamente: a incapacidade do tutor dar apoio de uma forma rápida e constante, a falta de diagnóstico de alunos e, sobretudo, a falta de um acompanhamento mais ou menos personalizado dos estudantes, resultante do enorme crescimento verificado nos cursos superiores de EaD e dos MOOCs, associado a um aumento de tutores não proporcional, que contribui para um apoio deficiente aos alunos, neste contexto.

O objetivo deste artigo foi compreender os novos desafios da EaD no ensino superior on-line, nomeadamente a programação e uso de Chatbots, bem como as razões e causas para sua operacionalidade. Podemos afirmar que, em suma, os resultados identificados destacaram as vantagens da programação e uso dos Chatbots nos cursos de EaD, pois a sua programação é acessível e intuitiva, considerando os aplicativos e interfaces existentes. Existem inúmeras plataformas disponíveis no mercado, para esse fim, e foi evidente que esta tecnologia pode contribuir de forma significativa para uma melhoria na comunicação e na relação, entre os tutores e os estudantes.

\section{Referências}

ALVES, D. G.; CABRAL, T. D.; COSTA, R. M. E. M. Ambientes virtuais para educação a distância: uma estrutura de classificação e análise de casos. Cadernos do IME - Skie Informática, Rio de Janeiro, v. 14, p. 2, jun. 2003.

AMARILLA, P. F. Educação a distância: uma abordagem metodológica e didática a partir dos ambientes virtuais. Educação em Revista, Belo Horizonte, v. 27, n. 2, p. 41-72, ago. 2011.

ANDERSON, T. Theory and Practice of Online Learning. 2. ed. Athabasca: AU Press, 2008.

ARETIO, L. G.; CORBELLA, M. R.; FIGAREDO, D. D. De la educación a distancia a la educación virtual. Barcelona: Ariel, 2007. 
BARROS, D. M. V.; REIS, V. A função tutorial na formação continuada docente. Revista Iberoamericana de Educación a Distancia, v. 1, p. 10-20, 2009.

BELLONI, M. L. Educação a distância. Campinas: Autores Associados, 2008.

BIDAISEE, S. 6 ways to increase MOOC retention. 2017. Disponível em: <https://www.ecampusnews.com/2017/09/19/7-ways-increase-mooc-retention/>. Acesso em: 13 set. 2018.

COSTA, E. G. Tendências contemporâneas em educação superior a distância no mundo e no Brasil. Espacio Abierto - Cuaderno Venezolano de Sociologİa, Maracaibo, Venezuela, v. 25, n. 3, p. 265-289, set. 2016.

COYNE, A. Meet Genie, Deakin Uni's virtual assistant for students. 2017. Disponível em: <https://www.itnews.com.au/news/meet-genie-deakin-unis-virtual-assistant-for-students-453230>. Acesso em: 30 maio 2018.

DOWNES, S. E-Learning 2.0 In Development. Research Innovations in Learning Conference. San Jose, California: Brandon Hall Associates, 2007.

FERREIRA, A. B. H. Novo dicionário da língua portuguesa. 2. ed. rev. e aum. Rio de Janeiro: [s.n.], 1986.

GARRISON, D. R. Understanding distance education: a framework for the future. London: Routledge, 1989.

GARRISON, R.; ANDERSON, T. E-Learning in the 21st Century: a framework for research and practice. London: Routledge, 2003.

GOEL, A. K.; POLEPEDDI, L. Jill Watson: a Virtual Teaching Assistant for Online Education. Atlanta, Georgia, USA: Design \& Intelligence Laboratory, School of Interactive Computing, Georgia Institute of Technology, 2016.

GOMES, C. Chatbot: entenda tudo sobre o assunto. 2017. Disponível em: <http://blog.simply. com.br/chatbot/>. Acesso em: 30 maio 2018.

HOLLANDS, F. M.; TIRTHALI, D. MOOCs: Expectations and Reality. [S.l.]: Columbia University, 2014.

KHALIL, H.; EBNER, M. MOOCs Completion Rates and Possible Methods to Improve. In: WORLD CONFERENCE ON EDUCATIONAL MULTIMEDIA, HYPERMEDIA AND TELECOMMUNICATIONS. Proceedings... Chesapeake, 2014.

KIRNER, C.; SISCOUTTO, R. Realidade virtual e aumentada: conceitos, projeto e aplicações. Petrópolis, RJ: [s.n.], 2007.

KOEHLER, M. J.; MISHRA, P. What Is Technological Pedagogical Content Knowledge? Contemporary Issues in Technology and Teacher Education, Michigan, USA, v. 9, n. 1, p. 60-70, 2009.

LACITY, M. et al. Reimagining the University at Deakin: An IBM Watson Automation Journey Research on Business Services Automation. Deakin, Australia: Deakin University, 2017. p. 1-26.

LEAL, J.; GOUVEIA, L. B. MOOC: qual o papel na reconceptualização na universidade? In: CONGRESSO INTERNACIONAL PSICOLOGIA, EDUCAÇÃO E CULTURA, 2. Anais... [S.l.: s.n.], 2015. p. 197-206. 
LEITE, L. S.; BRAGLIA, I.; PEREIRA, A. T. C. Realidade virtual na educação à distância de projeto de arquitetura. In: Congresso Internacional de Ergonomia e Usabilidade de Interfaces Humano-Computador, 11, 15 a 19 de maio de 2011. Anais... Manaus, 2011.

LEONHARDT, M. D.; CASTRO, D. D.; TAROUCO, L. M. R. ELEKTRA: um Chatterbot para uso em ambiente educacional. Revista Novas Tecnologias na Educação, Porto Alegre, v. 1, n. 2, p. 1-11, set. 2003.

LIPKO, H. Meet Jill Watson: Georgia Tech's first AI teaching assistant. 2016. Disponível em: <https://pe.gatech.edu/blog/meet-jill-watson-georgia-techs-first-ai-teaching-assistant>. Acesso em: 18 jul. 2018.

LUSTOSA, V. G.; ALVARENGA, R. O estado da arte em inteligência artificial. Revista Digital da CVA - Ricesu, Brasília, DF, v. 2, n. 8, p. 2-10, set. 2004.

MACHADO, L. D.; MACHADO, E. C. O papel da tutoria em ambientes de EAD. 2004. Disponível em: <http://www.abed.org.br/congresso2004/por/htm/022-tc-a2.htm>. Acesso em: 24 set. 2018.

MARTINS, V. F.; GUIMARÃES, M. P. Desafios para o uso de Realidade Virtual e Aumentada de maneira efetiva no ensino. [S.l.: s.n.], 2012.

MOORE, M. G.; ANDERSON, W. G. Handbook of distance education. NJ: Mahwah: Lawrence Erlbaum Associates, 2003.

MORGADO, L. Os novos desafios do tutor a distância: o regresso ao paradigma da sala de aula. Perpectivas em Educação. Lisboa: Universidade Aberta, 2003.

MORGAN, B. How Chatbots will Transform Customer Experience: an Infographic. 2017. Disponível em: <https://www.forbes.com/sites/blakemorgan/2017/03/21/how-chatbots-will-transform-customer-experience-an-infographic/\#994d2c87fb4a>. Acesso em: 2 jul. 2018.

MULDOWNEY, O. Chatbots: an introduction and easy guide to making your own. Dublin, Ireland: Curses \& Magic, 2017.

NOFFS, N. A.; TORRES, B. S. Tutoria: uma prática educativa para a inclusão social. 2014. Disponível em: <https://direcionalescolas.com.br/tutoria-uma-pratica-educativa-para-a-inclusao-social/>. Acesso em: 6 jul. 2018.

NUNES, I. B. Capítulo 1: A história da EAD no mundo. In: LITTO, F.; FORMIGA, M. Educação a Distância: o estado da arte. São Paulo: Pearson Education do Brasil, 2009. p. 2-8.

PEREIRA, A. et al. Modelo pedagógico virtual da Universidade Aberta: para uma universidade do futuro. Lisboa: Universidade Aberta, 2007.

RUSSELL, S.; NORVIG, P. Artificial Intelligence - a Modern Approach. Englewood Cliffs, New Jersey: Prentice Hall, 1995.

SANGRÀ, M. et al. Hacia una definición inclusiva del e-learning. Barcelona: eLearn Center, UOC, 2011.

SCHAPPO, V. Chatbot: o que é, quais são as suas as vantagens e como usar na sua empresa. 2017. Disponível em: <https://resultadosdigitais.com.br/blog/chatbot/>. Acesso em: 2 jul. 2018. 
SRDANOVIC, B. Chatbots in Education: Applications of Chatbot Technologies. 2017. Disponível em: <https://elearningindustry.com/chatbots-in-education-applications-chatbot-technologies $>$. Acesso em: 8 jul. 2018.

WALLACE, E.; ICE , P.; GIBSON , A. M. Comprehensive Assessment of Student Retention in Online Learning Environments. Online Journal of Distance Learning Administration, Georgia, USA, v. 14, n. 1, 2011. Disponível em: <https://eric.ed.gov/?id=EJ921846>. Acesso em: 8 jul. 2018.

ZUMSTEIN, D.; HUNDERTMARK, S. Chatbots - an interactive technology for personalized communication, transactions and services. IADIS International Journal on WWW/Internet, Lucerne, Switzerland, v. 15, n. 1, p. 96-109, Nov. 2017. 